Résumés des conférences et travaux

Problèmes d'ecdotique des documents diplomatiques médiévaux

\title{
Sébastien Barret
}

\section{OpenEdition}

\section{Journals}

Édition électronique

URL : https://journals.openedition.org/ashp/3055

DOI : 10.4000/ashp.3055

ISSN : 1969-6310

Éditeur

Publications de l'École Pratique des Hautes Études

Édition imprimée

Date de publication : 1 septembre 2019

Pagination : 257-259

ISSN : 0766-0677

Référence électronique

Sébastien Barret, «Problèmes d'ecdotique des documents diplomatiques médiévaux », Annuaire de I'École pratique des hautes études (EPHE), Section des sciences historiques et philologiques [En ligne], 150 | 2019, mis en ligne le 11 juin 2019, consulté le 06 juillet 2021. URL : http:// journals.openedition.org/ashp/3055; DOI : https://doi.org/10.4000/ashp.3055 


\title{
PROBLÈMES D'ECDOTIQUE DES DOCUMENTS DIPLOMATIQUES MÉDIÉVAUX
}

\author{
Chargé de conférences : M. Sébastien BARRET
}

Programme de l'année 2017-2018 : L'annotation historique des actes à l'ère numérique.

Les conférences de l'année universitaire 2017-2018 ont été tenues en 12 séances entre le 9 novembre et le 24 mai, les $2^{\mathrm{e}}$ et $4^{\mathrm{e}}$ jeudis normalement, à quelques irrégularités près. Elles se sont basées sur le travail qui avait été fourni en amont, lors de l'année 2016-2017, durant laquelle, notamment, des bases méthodologiques et théoriques avaient été fournies, qui n'ont pas été reprises au-delà d'un rappel initial et de retours ponctuels. En revanche, il a été fait retour sur l'un ou l'autre sujet qui avait été déjà abordé, pour l'approfondir. Les modifications mentionnées ci-dessous dans le programme initial ont mené au choix de décaler encore les questions les plus purement techniques vers la troisième année de conférences, où l'aspect purement numérique de la question doit être abordé de front, tant en ce qui concerne les méthodes que leur mise en œuvre sous la forme de projets d'édition. Ceci étant, une ample place a toujours été ménagée aux " nouvelles technologies », tant dans les questions posées que pour les réponses à y apporter.

L'on a tout d'abord consacré un groupe de séances aux questions liées à la description des documents, et notamment à leur description matérielle. Si cette dernière a toujours fait partie, au moins a minima, des protocoles suivis par les éditeurs de textes - ceci, du reste, depuis les travaux des érudits de l'époque moderne -, la question est renouvelée depuis quelques années. En effet, les questions liées à la matérialité des documentations anciennes ont occupé de plus en plus de place dans les interrogations des historiens. Il est donc logique que ces intérêts renouvelés puissent se voir, au moins potentiellement, reflétés dans les pratiques éditoriales. De plus, c'est aussi l'un des domaines où les technologies numériques et les possibilités qu'elles offrent peuvent faire sentir leur impact.

Il est de tradition de donner, à l'occasion du tableau de la tradition, les dimensions d'un document et de l'éventuel repli servant au scellage, ainsi qu'une brève description du sceau. Au cours même de l'édition du document, notamment pour les plus anciens, des moyens typographiques peuvent être utilisés pour représenter certaines des caractéristiques considérées comme importantes au niveau visuel, telles que l'emploi de lettres allongées, d'un chrismon ou autre invocation symbolique, d'une ruche de chancellerie, d'une croix de souscription... Des éditions plus spécifiquement tournées vers les documents originaux ont pu s'intéresser, dans leur commentaire, de plus près aux aspects matériels, notamment paléographiques et « codicologiques » (dans le sens de la « codicologie du document d'archives » évoquée par Paul Bertrand ${ }^{1}$ ). L'on s'est d'abord interrogé sur les éléments susceptibles d'être décrits et sur la manière

1. P. Bertrand, « Une codicologie du document d'archives existe-t-elle? », dans Gazette du livre médiéval, t. 54, 2009, p. 10-18 [en ligne] https://www.persee.fr/doc/galim_0753-5015_2009_num_54_1_1797 (consulté le 30.03.2019). 
de le faire. La question est, au fond, plus complexe qu'on pourrait le penser si l'on cherche à prendre en compte, au moins de manière théorique, tout ce qui pourrait être descriptible - par exemple, l'épaisseur du parchemin ou du papier, son état de conservation, les taches, les trous, la réglure, les piqûres (qu'elles soient liées à la réglure ou non; faut-il en décrire la forme ou celle du poinçon?), l'épaisseur du ruban d'écriture, des lettres, les caractéristiques morphologiques de ces dernières, les interlignes, les marges... Finalement, énormément de choses pourraient être prises en compte. Si l'on considère les capacités considérables de traitement des données offertes par les technologies numériques - ne serait-ce qu'en matière de rédaction et d'hébergement de textes dont la longueur n'est plus un problème technique -, l'on peut facilement être tenté d'aller très loin, y compris pour des documents pour lesquels l'on n'aurait pas pensé, il y a encore quelques années, mettre de grands frais dans une quelconque description. Sur la base d'exemples choisis, l'on s'est d'abord interrogé en commun sur l'identification des éléments qui pourraient être pris en compte, puis sur l'intérêt de leur commentaire et la place éventuelle de celui-ci, en relation avec les ensembles documentaires considérés, l'intérêt de leur édition, ses spécificités par rapport à celle d'autres documents, et les intentions éventuelles de l'éditeur; ceci en se mettant « en situation » de manière bien évidemment fictive, afin de multiplier les angles, et en considérant tant les documents en « feuilles simples » que les codices.

Un autre bloc thématique a concerné la manière de décrire et de commenter ce que l'on peut appeler grossièrement le fond des documents. Il est notamment d'usage, quand on édite, de donner une analyse en tête, et éventuellement de commenter plus avant dans la dissertation critique - sans oublier les éléments synthétiques donnés en introduction. C'est un point à la fois évident, très visible et très délicat. Il représente en effet souvent le premier (voire, parfois, le seul) contact de l'utilisateur d'une édition d'actes avec le contenu de tel ou tel document ou groupe de documents. Au-delà du débat récurrent entre éditeurs de textes diplomatiques sur l'opposition « analyse brève / analyse approfondie ", l'on a cherché, sur la base d'exercices pratiques, à illustrer les problèmes qui peuvent se poser. Le premier est de savoir dans quel degré de détail entrer et, surtout, de déterminer un degré de détail qui permettra de rester équilibré dans le rendu, non seulement d'un document particulier, mais aussi dans celui de l'ensemble édité. En effet, trop insister dans l'analyse sur certains aspects du document et non sur d'autres risque de fausser la perception qu'en aura le lecteur, notamment sur ce qu'il pourra encore découvrir lui-même dans le texte; et cet effet se retrouvera au niveau du corpus entier si l'éditeur ne prend pas garde à une certaine régularité. Il peut, notamment, être tentant de dissimuler une ignorance ou une incompréhension de détail en passant sous silence l'un ou l'autre élément mystérieux; mais il vaut mieux, au contraire, ne pas hésiter à le mentionner, quitte à laisser un mot difficile à comprendre dans sa langue d'origine. Ce dernier point a, du reste, fait l'objet de réflexions renouvelées, après avoir déjà été abordées lors du cycle 2016-2017. En effet, l'opération de traduction, en l'espèce souvent du latin ou de l'ancien français vers le français contemporain, peut produire un fâcheux effet de distorsion. L'on avait constaté il y a un an que cela pouvait faire croire à l'identification d'une personne derrière le simple passage de son nom en une langue familière; pareillement, il faut ici 
se souvenir que la réalité désignée par l'évolution vernaculaire d'un mot latin n'est pas forcément la même que celle du mot d'origine - sans même parler des différences synchroniques. Par exemple, l'on connaît les grandes difficultés que pose un terme tel que villa; symétriquement, le terme français de « finage » peut être trompeur s'il est utilisé abusivement. Là encore, un délicat équilibre doit être tenu entre la prise de décision, nécessaire, et la prudence, indispensable.

Du fait de besoins exprimés par les auditeurs, un détour a été entrepris par la critique du faux. Celle-ci, pilier des études de diplomatique médiévale et des éditions critiques depuis leurs débuts, fait en effet légitimement partie de ce que l'on peut qualifier de commentaire, même si l'on peut estimer à bon droit que c'est un commentaire particulier. Ce qui la rend, au fond, pertinente pour le thème des conférences, c'est qu'elle se conçoit aujourd'hui moins que jamais comme simple méthode d'élimination de documents défectueux. Le faux est un objet d'étude au même titre que le vrai, chargé de sens et de potentiel analytique. En conséquence, la reconnaissance et l'analyse du faux impliquent naturellement, au-delà de son identification, d'en reconstituer autant que possible le contexte et la genèse; ces derniers faisant partie de ce qui peut être légitimement discuté par l'éditeur, au même titre que les éléments donnés, souvent en introduction d'une édition, sur les chancelleries, scriptoriums, ateliers d'écriture... ayant produit les actes édités. Et de fait, les exemples évoqués (actes ou lettres douteux de Louis le Pieux ou Sanche le Grand par exemple) ont permis d'aborder plusieurs situations, tout d'abord en termes de transmission documentaire : les démarches ne sont pas les mêmes selon que l'on est en présence d'un pseudo-original, d'une copie médiévale ou d'une transcription moderne d'un document suspect. C'est peut-être encore plus important que dans le cas de l'édition d'un acte vrai : en effet, l'étude détaillée, quand elle est possible, de la tradition d'un faux peut être très éclairante sur ses origines. Elle peut aussi déboucher sur des hypothèses qui, tout en reconnaissant que le document n'est pas authentique, n'en font pas forcément une forgerie. Dans les cas les plus complexes, la transmission d'une charte peut très bien passer par l'association d'étapes frauduleuses et de moments honnêtes (copie de bonne foi d'un faux, mais aussi éventuellement ajout de couches d'interpolations sur un acte déjà douteux, par exemple).

Au cours de ces séances, des allusions aux questions d'encodage qui seront abordées de manière plus développée au cours de l'année 2018-2019 ont d'ores et déjà été faites. Les différents thèmes ont été abordés de manière pratique, à partir de cas concrets et de travaux récents ou en train de se faire, apportés par le chargé de conférences ou, à l'occasion, par les auditrices et auditeurs. L'on a ainsi pu évoquer, à l'appui des différents objets d'étude, des actes privés des $\mathrm{IX}^{\mathrm{e}}$ ou $\mathrm{X}^{\mathrm{e}}$ siècles, des documents impériaux carolingiens ou des chartes royales du XI ${ }^{\mathrm{e}}$ siècle; des documents de gestion, des registres de la fin du Moyen Âge; ou encore des cartulaires du Moyen Âge central. 